Note

\title{
Improved Method for the Analysis of Ascorbic Acid in Plasma by High-Performance Liquid Chromatography with Electrochemical Detection
}

\author{
Keizo Umegaki, Kikuko Inoue, Nozomi Takeuchi, \\ and Mitsuru HigucHI
}

The National Institute of Health and Nutrition, Shinjuku-ku, Tokyo 162, Japan

(Received October 19, 1993)

\begin{abstract}
Summary We improved the analytical method for the detection of ascorbic acid in plasma by high-performance liquid chromatography (HPLC) with an electrochemical detector (ECD) to be more selective and rapid than the protocol previously used. Main improvements are as follows. Applied potential of ECD to obtain the maximal response for ascorbic acid was $+450 \mathrm{mV}$ versus $\mathrm{Ag} / \mathrm{AgCl}$, but it was reduced to +350 $\mathrm{mV}$. In that condition, uric acid did not respond to ECD, and only ascorbic acid was detected. EDTA contained in sample extraction/stabilizing solution gave the peak after the ascorbic acid. The addition of EDTA $(0.2 \mathrm{mM})$ to the mobile phase eliminated the EDTA peak. These two improvements gave the chromatogram in which the peak that appeared from the plasma sample was only ascorbic acid, and shorten the sample run time. Ascorbic acid in plasma was unstable even though the plasma was treated with methanol/EDTA: it decreased from $1.5 \mathrm{~h}$ at $4^{\circ} \mathrm{C}$. However, the treated sample, which was placed at $-14^{\circ} \mathrm{C}$ until the analysis was performed, gave the reliable ascorbic acid value at least up to $6 \mathrm{~h}$. The data obtained from the HPLC-ECD method was consistent with those from the hydrazine method.
\end{abstract}

Key Words ascorbic acid, uric acid, plasma, electrochemical detection, high-performance liquid chromatography

Hydrazine method (colorimetric method) is a widely used method for the analysis of plasma ascorbic acid (1). However, the method is claimed to be insensitive and inferior in specificity (2). The methods using high-performance liquid chromatography (HPLC) with fluorometric (3), ultraviolet (4-7), or electrochemical detector (ECD) (7-12) has been developed. Among the methods, the HPLC-ECD method is extremely sensitive and selective for ascorbic acid without derivatization of ascorbic acid (2). Thus, several separating conditions using different columns and solvent systems have been reported in the method. In plasma, 
uric acid is present about 5 times as much as ascorbic acid, and reacts with ECD at the similar electrode potential of ascorbic acid $(7,11,12)$. In addition, uric acid and ascorbic acid have close retention time in the chromatogram of HPLC, especially in a short run time. Thus, it is important to êliminate the interference of uric acid for the detection of ascorbic acid in plasma by the HPLC-ECD. In this study, we modified the method of HPLC-ECD reported by Washko et al. (10) to be more selective, and rapid for ascorbic acid without interference of uric acid.

Materials and methods. Preparation of standard solution: Ascorbic acid and uric acid were purchased from Wako Pure Chemical Co. Ltd., Tokyo. Ascorbic acid was dissolved in $60 \%$ methanol (volume \%) in water containing $1 \mathrm{~mm}$ ethylenediaminetetraacetic acid (EDTA), diluted with the $60 \%$ methanol/EDTA solution to about $1 \mu \mathrm{g} / \mathrm{ml}$, and stored at $-80^{\circ} \mathrm{C}$ until use. Uric acid was dissolved in distilled water, and diluted with the $60 \%$ methanol/EDTA solution.

Preparation of plasma sample: Peripheral blood $(10 \mathrm{ml})$ was taken from antecubital vein of healthy volunteers into a heparinized tube. The blood was immediately centrifuged at $1,500 \times g$ for $15 \mathrm{~min}$ at $4^{\circ} \mathrm{C}$ and the plasma was prepared. For the analysis of ascorbic acid by colorimetric method, $0.5 \mathrm{ml}$ of the plasma was immediately mixed with an equal volume of $30 \%$ methaphosphoric acid, and the mixture was stored at $-80^{\circ} \mathrm{C}$ until the analysis was performed. For the analysis of ascorbic acid by HPLC-ECD, $30 \mu \mathrm{l}$ of the plasma was immediately mixed with two volumes of $90 \%$ methanol in water saturated with EDTA (an extraction/stabilizing solution) in a microtube, placed on ice for $10-15 \mathrm{~min}$, then centrifuged at $12,000 \times g$ for $5 \mathrm{~min}$ at $4{ }^{\circ} \mathrm{C}$. The resulting supernatant $(1-5 \mu \mathrm{l})$ was injected into HPLC-ECD.

Analysis of ascorbic acid: HPLC-ECD system used for the analysis of ascorbic acid was as follows: pump, sigma 871 (IRICA, Kyoto); sample injector, sigma 80 (IRICA); ECD (applied potential, $+350 \mathrm{mV}$ versus $\mathrm{Ag} / \mathrm{AgCl}$ ), sigma 875 (IRICA); column, QC Pack C18 $(4.5 \times 250 \mathrm{~mm}$, IRICA) or TSK gel ODS $120 \mathrm{~A}$. $(4.5 \times 250 \mathrm{~mm}$, Toyo Soda, Tokyo). The mobile phase was: $50 \mathrm{~mm}$ sodium phosphate, $50 \mathrm{~mm}$ sodium acetate, $189 \mu \mathrm{M}$ dodecyltrimethylammonium chloride (Kanto Chemical Co. Inc.), $36.6 \mu \mathrm{M}$ tetraoctylammonium bromide (Sigma, St. Louise Mo., U.S.A.), 0.2 mM EDTA, 20\% methanol in water, $\mathrm{pH} 4.8$. The dodecyltrimethylammonium chloride was dissolved in the methanol first, and the $\mathrm{pH}$ of the final solution was adjusted to 4.8 with phosphoric acid as reported by Washko et al. (10). The flow rate was set at $1 \mathrm{ml} / \mathrm{min}$.

As the conventional method of ascorbic acid analysis, the (dinitrophenyl) hydrazine method reported by Omaye et al. (1) was used.

Statistical analysis: The statistical analyses were done by using a computer program (Stat Flex) (JIP, Tokyo).

Redults and discussion. The applied potential of ECD for ascorbic acid reported was not consistent (7-12). Therefore, we examined the applied potential which gave the highest peak of ascorbic acid in the ECD used. The potential was about $+450 \mathrm{mV}$ versus $\mathrm{Ag} / \mathrm{AgCl}$. Then potential of ECD was set at $+450 \mathrm{mV}$, and 
plasma sample was subjected to the HPLC-ECD. The mobile phase was the original components reported by Washko et al. (10): $50 \mathrm{~mm}$ sodium phosphate, 50 $\mathrm{mm}$ sodium acetate, $189 \mu \mathrm{M}$ dodecyltrimethylammonium chloride, $3.66 \mu \mathrm{M}$ tetraoctylammonium bromide, $30 \%$ methanol in water, $\mathrm{pH} 4.8$. In that condition, uric acid greatly interfered with ascorbic acid detection. The retention time was 2.89 min with uric acid and $3.0 \mathrm{~min}$ with ascorbic acid. When the composition of the solvent was changed, the separation of ascorbic acid and uric acid was slightly improved. But the interference of uric acid was still observed. Then, we applied 1 ng of ascorbic acid or uric acid to the HPLC-ECD with the different applied potential of ECD and explored the existence of applied potential at which ascorbic acid specifically reacts. As shown in Fig. 1, the reactivity of ascorbic acid to ECD was different from that of uric acid. When the applied potential of ECD was set at $+350 \mathrm{mV}$, uric acid did not react to ECD and ascorbic acid reacted at $80 \%$ of the maximum level. Thus, we concluded that the applied potential of ECD was specific for ascorbic acid. As reported (7-12), the applied potential is different among the ECDs. Therefore, we should check the suitable applied potential of an available ECD by subjecting ascorbic acid and uric acid.

When the applied potential of ECD was set at $+350 \mathrm{mV}$ and standard ascorbic acid was injected into the HPLC-ECD, a peak was detected just after the ascorbic acid peak (Fig. 2a). The peak became higher as the injection volume of the standard or plasma sample increased. The peak was derived from EDTA in the sample extraction/ stabilizing solution as reported by Dhariwal et al. (12). By the addition of EDTA $(>0.2 \mathrm{~mm})$ to the mobile phase, no EDTA peak appeared and the base line was stabilized in the chromatogram (Fig. 2b). When plasma sample

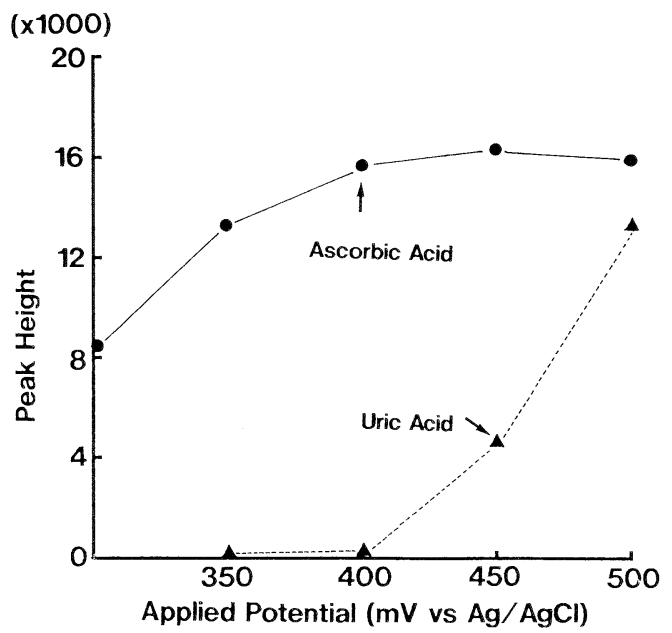

Fig. 1. Response of ascorbic acid and uric acid at different applied potential of ECD. One nanogram per five microliters of ascorbic acid or uric acid was injected into HPLC with ECD at different applied potential (versus $\mathrm{Ag} / \mathrm{AgCl}$ ). 


\section{Standard}

a)

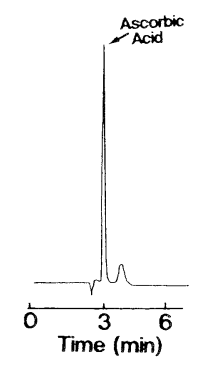

Solvent $\rightarrow$ Original

$\mathrm{ECD} \quad \rightarrow+350 \mathrm{mv}$ b)

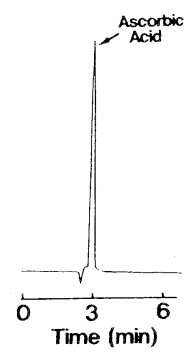

Original + EDTA

$+350 \mathrm{mV}$
Plasma Sample

c)

d)

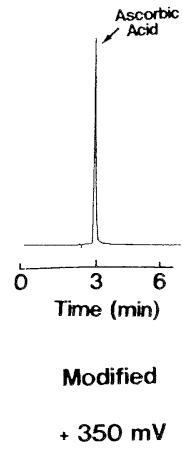

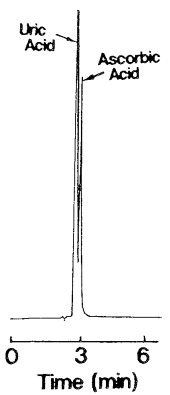

Modified

$+450 \mathrm{mV}$

Fig. 2. Typical chromatogram of the standard ascorbic acid and plasma sample. Ten microliters of standard ascorbic acid $(0.5 \mu \mathrm{g} / \mathrm{ml})$, and $1 \mu \mathrm{l}$ of plasma sample treated with methanol/EDTA were injected into HPLC-ECD. The mobile phase in a) was the original component reported by Washko et al. (10) and that in b) was added with EDTA $(0.2 \mathrm{~mm})$. The mobile phase of c) and d) was the component modified in this study. The applied potential of ECD in a) to c) was $350 \mathrm{mV}$, but that in d) was $450 \mathrm{mV}$.

was injected into the HPLC-ECD system, the peak detected was only ascorbic acid. This could shorten the sample run time of HPLC-ECD. To decrease the column back pressure and the retention time of ascorbic acid, we further modified the solvent composition. That was decreasing the content of methanol from 30 to $20 \%$ and increasing the concentration of tetraoctylammonium bromide, an ion-pair reagent, from 3.66 to $36.6 \mu \mathrm{M}$. The typical chromatogram of the plasma sample using the modified solvent is shown in Fig. 2c. As expected, when the applied potential of ECD increased from 350 to $450 \mathrm{mV}$, a marked interference of uric acid was observed (Fig. 2d).

It has been reported that ascorbic acid in plasma was unstable even though it was treated with stabilizing solution such as methaphosphoric acid and methanol/ EDTA $(6,8,11,12)$. The stability of ascorbic acid in standard solution and deproteinized plasma treated with the methanol/EDTA was examined in the improved HPLC-ECD system. The standard solution and the plasma sample were placed in either $25^{\circ} \mathrm{C}$ (at room temperature), $4^{\circ} \mathrm{C}$ (in refrigerator), or $-14^{\circ} \mathrm{C}$ (in freezer), then injected into the HPLC-ECD. The standard ascorbic acid was stable up to $3 \mathrm{~h}$ at $25^{\circ} \mathrm{C}$ and up to several weeks at $-14^{\circ} \mathrm{C}$ (Fig. 3a). In contrast, ascorbic acid in plasma sample was unstable: it could hardly be detected at $2.5 \mathrm{~h}$ at $25^{\circ} \mathrm{C}$ and decreased by $20 \%$ at $2.5 \mathrm{~h}$ at $4^{\circ} \mathrm{C}$ (Fig. $3 \mathrm{~b}$ ). The result was consistent with the report of Dhariwal et al. (12). However, the ascorbic acid of the plasma sample 
a) Standard

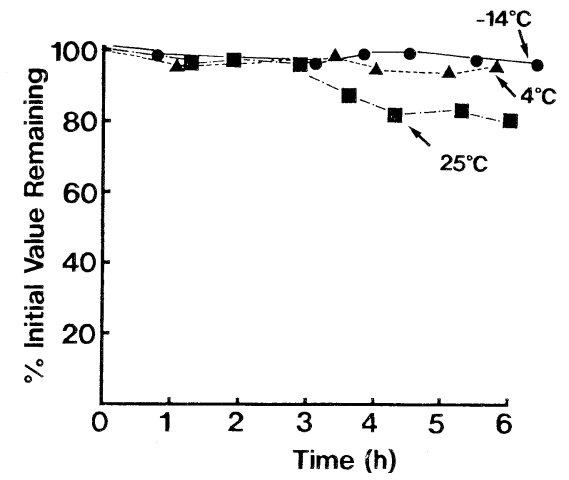

b) Plasma Sample

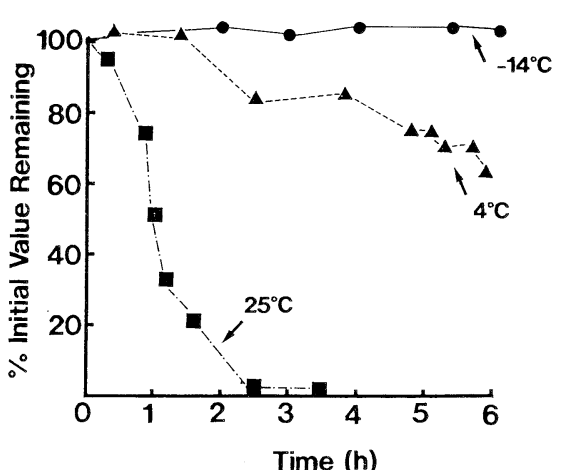

Fig. 3. Stability of ascorbic acid in standard solution and plasma sample. Five microliters of standard ascorbic acid $(1 \mu \mathrm{g} / \mathrm{ml})$, and $2 \mu \mathrm{l}$ of plasma sample treated with methanol/EDTA were placed at 25,4 , or $-14^{\circ} \mathrm{C}$ for the indicated time and injected into HPLC-ECD.

placed at $-14^{\circ} \mathrm{C}$ did not decrease up to $6 \mathrm{~h}$. To place the plasma sample at $-14^{\circ} \mathrm{C}$ was convenient; the sample did not freeze at the temperature due to the presence of methanol, and could be immediately injected into HPLC. Thus, the best way to do the reliable analysis of plasma ascorbic acid might be to place the plasma sample at $-14^{\circ} \mathrm{C}$ until the analysis is performed and to inject the sample into HPLC-ECD immediately after the preparation.

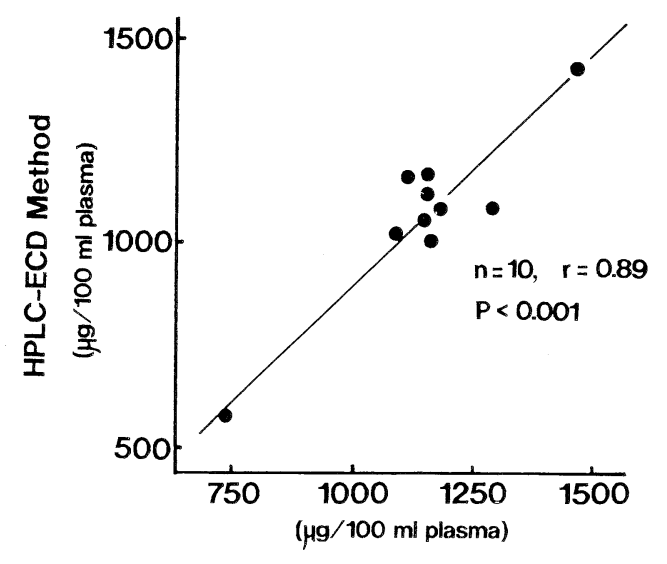

Hydrazine Method

Fig. 4. Relationship of plasma ascorbic acid concentrations between hydrazine method and HPLC-ECD method. Plasma samples were obtained from 10 healthy human subjects, and their ascorbic acid concentrations were analyzed by hydrazine method or HPLC-ECD method modified in this study. 
Ascorbic acid concentrations in plasma obtained from 10 subjects were analyzed by both the colorimetric method and the HPLC-ECD method improved in this study. The data obtained from the two methods were significantly correlated (Fig. 4). The detection limit of ascorbic acid in the HPLC-ECD method was less than $0.1 \mathrm{ng}$ of ascorbic acid, which corresponds to $0.01 \mu \mathrm{l}$ of plasma. Therefore, it is possible to analyze ascorbic acid in blood obtained from finger. In fact, we analyzed ascorbic acid using $5 \mu 1$ of whole blood from first finger, and got reliable data. The method using the small amount of blood obtained from finger could be suitable for continuous monitoring of ascorbic acid in blood such as on exercises.

\section{REFERENCES}

1) Omaye, S. T., Turnbull, J. D., and Sauberlich, H. E. (1979): Selected methods for the determination of ascorbic acid in animal cells, tissue and fluids. Method Enzymol., 62, 3-11.

2) Washko, P. W., Welch, R. W., Dhariwal, K. R., Wang, Y., and Levine, M. (1992): Ascorbic acid and dehydroascorbic acid analyses in biological samples. Anal. Biochem., 204, 1-14.

3) Lopez-Anaya, A., and Mayersohn, M. (1987): Ascorbic and dehydroascorbic acids. simultaneously quantified in biological fluids by liquid chromatography with fluorescence detection, and comparison with a colorimetric assay. Clin. Chem., 33, 18741878.

4) Kishida, E., Nishimoto, Y., and Koji, S. (1992): Specific determination of ascorbic acid with chemical derivatization and high-performance liquid chromatography. Anal. Chem., 64, 1505-1507.

5) Ziegler, S. J., Meier, B., and Sticher, O. (1987): Rapid and sensitive determination of dehydroascorbic acid in addition to ascorbic acid by reversed-phase high-performance liquid chromatography using a post-column reduction system. J. Chromatogr., 391, 419-426.

6) Liau, L. S., Lee, B. L., New, A. L., and Ong, C. N. (1993): Determination of plasma ascorbic acid by high-performance liquid chromatography with ultraviolet and electrochemical detection. J. Chromatogr., 612, 63-70.

7) Cammack, J. (1991): Simultaneous high-performance liquid chromatographic determination of ascorbic acid and dehydroascorbic acid in biological samples. J. Chromatogr., 565, 529-532.

8) Kutnink, M. K., Hawkes, W. C., Schaus, E. E., and Omaye, S. T. (1987): An internal standard for the unattended high-performance liguid chromatographic analysis of ascorbic acid in blood components. Anal. Chem., 166, 424-430.

9) Behrens, W. A., and Madere, R. (1987): A high sensitive high-performance liquid chromatography method for the estimation of ascorbic and dehydroascorbic acid in tissues, biological fluids, and foods. Anal. Biochem., 165, 102-107.

10) Washko, P. W., Hartzell, W. O., and Levine, M. (1989): Ascorbic acid analysis using high-performance liquid chromatography with coulometric electrochemical detection. Anal. Biochem., 81, 276-282.

11) Nagy, E., and Degrell, I. (1989): Determination of ascorbic acid and dehydroascorbic 
acid in plasma and cerebrospinal fluid by liquid chromatography with electrochemical detection. J. Chromatogr., 497, 276-281.

12) Dhariwal, K. R., Hartzell, W. O., and Levine, M. (1991): Ascorbic acid and dehydroascorbic acid measurements in human plasma and serum. Am. J. Clin. Nutr., 54, 712-716. 